Para enlazar con este artículo / To link to this article:

https://doi.org/10.6035/MonTI.2020.12.14

Para citar este artículo / To cite this article:

Anssari-Naim, Saida. (2020) "Interpretación social y accesibilidad. Una propuesta de conexión disciplinar." En: Richart-Marset, Mabel \& Francesca Calamita (eds.) 2020. Traducción y Accesibilidad en los medios de comunicación: de la teoría a la práctica / Translation and Media Accessibility: from Theory to Practice. MonTI 12, pp. 418-439.

\title{
INTERPRETACIÓN SOCIAL Y ACCESIBILIDAD. UNA PROPUESTA DE CONEXIÓN DISCIPLINAR ${ }^{1}$
}

\author{
SAIDA ANSSARI-NAIM \\ saida.anssari@uv.es \\ Universidad de Valencia
}

\section{Resumen}

El objetivo de este estudio es ofrecer argumentos para entender la actividad propia de la interpretación social como parte del dominio genérico de la accesibilidad. Al hacerlo no ampliamos en realidad el concepto de accesibilidad, sino que reforzamos aquello que justifica el uso de este término, para diferenciar una parcela de actividad dentro de la práctica general de la traducción y la interpretación. Con el tema de la accesibilidad sucede que se ha manejado una etiqueta sin una delimitación conceptual previa de lo que se pretende designar por medio de ella. En este estudio nos ocupamos brevemente de esta tarea de aproximación conceptual, con el objetivo de incluir bajo el abanico de las prácticas de accesibilidad las labores concretas de un intérprete social.

Palabras clave: Interpretación social; Accesibilidad; Barreras comunicativas; Inmigración; Democracia.

\section{Abstract}

The aim of this study is to offer some arguments for the understanding of community interpreting as part of the generic domain of accessibility. By doing so, I do not actually

1. El presente estudio forma parte de las actividades financiadas por el proyecto del Ministerio de Ciencia, Innovación y Universidades FFI2017-84951-P: "Dimensiones metacognitivas en la adquisición, las alteraciones clínicas del lenguaje y la práctica interlinguaística: un enfoque pluridisciplinar". 
broaden the concept of accessibility, but I rather reinforce what justifies the use of this term, to differentiate a parcel of activity within the general practice of translation and interpreting. In what concerns accessibility, it seems that the label has been used with no prior conceptual delimitation of what it is finally intended to designate by it. In this study I briefly deal with the conceptual clarification, with the aim of including in the set of accessibility practices the specific task of community interpreting.

Keywords: Community Interpreting; Accessibility; Communicative barriers; Immigration; Democracy.

\section{Introducción}

La accesibilidad es un concepto que se inscribe dentro del proceso histórico en que se desarrollan las sociedades democráticas. En los orígenes ilustrados de las sociedades modernas, una noción transversal fue la de emancipación del individuo humano, esto es, nos referimos a aquello que lo hace autónomamente responsable sobre las decisiones que lo afectan. Esta emancipación individual es, en realidad, la base conceptual que permite el desarrollo de las democracias modernas. Remitir a los individuos la toma de decisiones éticas o políticas (también las económicas) requiere pensar antes en los individuos no como sujetos tutelados, sino como libres en la toma de estas decisiones. Si no es así, ¿cómo asignarles este tipo de responsabilidad? El concepto de emancipación no se plasmó un buen día en la constitución americana o con la revolución francesa, sino que se ha ido desarrollando progresivamente en los dos últimos siglos de la historia europea y mundial. Qué es lo que hace dependiente al individuo no es una pregunta que se haya podido resolver, sin embargo, con respuesta única, ni definitiva.

Si pensamos, por ejemplo, en términos de género, el reconocimiento de una dependencia que debía eliminarse ha sido, como es bien sabido, un largo proceso reivindicativo del movimiento feminista en el que, por supuesto, queda un largo camino por recorrer. Algo parecido podemos decir de la emancipación colonial, con una secuela de "efectos postcoloniales" que se viven y vivirán todavía durante un largo tiempo. El abanico de cuestiones que se pueden traer aquí a debate sería posiblemente interminable. Afecta a una pluralidad de aspectos tanto de las prácticas sociales como de la manera de reflexionar sobre ellas. Los estudios de traducción tampoco 
han quedado al margen del debate y, solo como una simple muestra, nos remitimos a la reformulación que Hurtado Albir (1990) nos propone sobre el concepto de fidelidad, que debe replantearse asignando al traductor el rol de agente dotado de autonomía en la gestión de su tarea y no como un mero sujeto subordinado. En esta misma línea se inscribe la concepción de la traducción como reescritura (Lefevere 1992), aspecto sobre el que volveremos más adelante.

La emancipación afecta por supuesto al individuo y sus derechos, pero tiene que ver al mismo tiempo con el entorno social con el que necesariamente este individuo interacciona, esto es, tiene que ver con otros sujetos humanos. La emancipación forma parte de un proceso mental de "autoconciencia" del ser humano que no puede formularse al margen de las relaciones de "empatía" respecto a otros individuos. Hay, según parece, bases neurofisiológicas que lo explican ${ }^{2}$. Por una razón de base antropológica profunda la emancipación o liberación individual no puede acabar de expresarse cuando me rodean sujetos esclavos o dependientes. Es la cuestión que ya planteaba Hegel en su conocida dialéctica del amo y el esclavo. Aunque pueda llevarme a engaño al respecto, en realidad no consigo ser más libre subordinando a otros sujetos humanos. Se trata de una visión errónea del verdadero poder social. Formulada la cuestión en otros términos, la emancipación está relacionada con el principio de "cooperatividad" en la gestión de la vida humana, en todas sus dimensiones. En este sentido, eliminar las barreras que imponen situaciones de dependencia, no debe considerarse como una simple labor filantrópica que los "no dependientes" realizan a favor de los "dependientes", sino que es algo que beneficia globalmente al cuerpo social.

2. Matusall (2013: 1): "Recently, several behavioral sciences became increasingly interested in investigating biological and evolutionary foundations of (human) social behavior. In this light, prosocial behavior is seen as a core element of human nature. A central role within this perspective plays the "social brain" that is not only able to communicate with the environment but rather to interact directly with other brains via neuronal mind reading capacities such as empathy. From the perspective of a sociologist, this paper investigates what "social" means in contemporary behavioral and particularly brain sciences. It will be discussed what "social" means in the light of social neuroscience and a glance into the history of social psychology and the brain sciences will show that two thought traditions come together in social neuroscience, combining an individualistic and an evolutionary notion of the "social." 
La gestión de nuestras relaciones con los otros se hace, sin embargo, cada vez más problemática en sociedades complejas postmodernas. La diversidad que las caracteriza puede considerarse una realidad de facto, cuando las comparamos por ejemplo con las sociedades existentes antes de que se desarrollara la globalización económica. Pero más importante incluso que esta diversidad de facto, es la progresiva toma de conciencia de esta diversidad. La conciencia de la diversidad se manifiesta de múltiples formas, afecta transversalmente a una pluralidad de situaciones: diversidad de las relaciones familiares, diversidad de género, de las prácticas alimentarias, diversidad de religión, de cultura y, por supuesto, también de lenguas. La accesibilidad es un concepto que visibiliza justamente la necesidad de gestionar la diversidad social. Esto afecta no solo a lo que podríamos considerar una diversidad que se va acrecentando en los tiempos actuales, sino también al reconocimiento de factores de diversidad social que han estado siempre presentes en las sociedades humanas, pero que no habían sido tenidos en cuenta o no lo habían sido en términos de inclusividad o cooperatividad. El caso del colectivo sordo resulta paradigmático en este sentido. Desde una visión patológica de este factor de la diversidad humana se ha ido pasando al reconocimiento de una identidad lingüístico-cultural para este colectivo (Sacks 1980), que permite su plena integración en las prácticas sociales, culturales y políticas. Queda, por supuesto, todavía mucho camino por recorrer también en este caso particular.

La accesibilidad, en definitiva, atiende problemas individuales de dependencia, pero sobre todo en la medida en que los individuos representan colectivos sociales con riesgo de exclusión, y que verían limitado por este motivo su contribución cooperativa a la vida social, lo que incluye por supuesto su contribución a la vida económica de un país. La accesibilidad es un término en proceso de ampliación conceptual, lo que se explica si tenemos en cuenta la indefinición y apertura del concepto de lo diverso y de lo otro. El término accesibilidad se usó originariamente con una lectura estrictamente física, relacionada con la eliminación de las barreras arquitectónicas. Pero queda claro -y resulta absolutamente legítimo- ampliar la esfera de significación del término a otros dominios de la dependencia, y especialmente al ámbito de los procesos comunicativos. Esto parece claro, si entendemos que la comunicación es la 
base fundamental de la actividad cooperativa humana. Nos referimos a esta cuestión específica en la siguiente sección de este estudio.

\section{Relaciones entre accesibilidad, comunicación y mediación intercódigo}

Aunque, como se acaba de señalar, el uso originario del término "accesibilidad" se refería fundamentalmente a la eliminación de barreras físicas que implican una limitación en el acceso de personas con problemas de movilidad a espacios tanto públicos como privados, su aplicación al ámbito comunicativo se ha hecho común en la actualidad. Alonso (2007: 19) incluye la comunicación, junto a otros ámbitos de aplicación del término como el urbanismo, la edificación y el transporte. La barreras físicas presentes en el uso originario del término tienen una obvia correspondencia en el ámbito comunicativo: se trata de las barreras asociadas a las diferencias de código lingüístico, entendido este último en el sentido más genérico del término, que incluye tanto diferencias de lengua, como diferencias de código expresivo dentro de una misma lengua.

La figura del traductor como sujeto que, también en un sentido genérico, salva este tipo de barreras comunicativas, adquiere el papel de agente mediador de la accesibilidad. Pero definir este último rol exige diferenciar una parcela de su actividad general como mediador intercódigo. En el siguiente gráfico se presenta un diagrama básico de conexiones entre accesibilidad y mediación intercódigo. Explicamos en lo que sigue tanto los subespacios disjuntos como el espacio de intersección, que se articula a su vez en dos niveles:

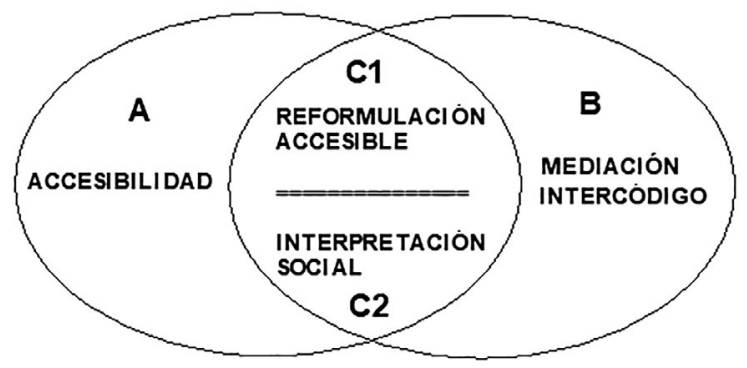

A. En el gráfico precedente podemos diferenciar, a la izquierda, un subdominio genérico de la accesibilidad disjunto respecto a la actividad comunicativa. 
Nos referimos fundamentalmente a la accesibilidad entendida en términos físicos. Ello no quiere decir, sin embargo, que la eliminación de barreras físicas no acabe siendo también una manera de facilitar los procesos comunicativos. La comunicación, como se ha dicho, es el medio fundamental para la acción cooperativa humana. Y hacer que los individuos se impliquen en la misma es el objetivo último de la accesibilidad.

B. Por mediación intercódigo nos referimos a todo proceso que transforma los mecanismos de codificación o descodificación de un texto preservando en lo esencial sus contenidos. La mediación intercódigo puede implicar cambio de lengua, cambio de medio expresivo, cambio de género discursivo, etc. Entendida así la cosa, existe también un subdominio de la mediación intercódigo disjunto respecto a la noción de accesibilidad, que se refleja en la parte derecha del gráfico. En términos muy generales toda actividad de mediación intercódigo supone eliminar barreras comunicativas, pero es obvio que no es este el sentido de la accesibilidad. La pregunta que cabe plantearse sería cuándo y por qué una actividad de mediación intercódigo puede conceptualizarse en términos de accesibilidad. Responder a esta pregunta es de manera central acotar el sentido de la accesibilidad. Este sentido tiene que ver fundamentalmente con lo ya formulado: la accesibilidad es el medio que permite la inclusión social en la acción cooperativa humana de determinados colectivos, que se verían privados del acceso a bienes y servicios en el caso de no verse facilitados los procesos comunicativos con operaciones de mediación intercódigo. La codificación en morse de un texto es una operación de mediación intercódigo, que hace posible su transmisión por vía telegráfica, pero obviamente esta operación nada tiene que ver con la accesibilidad. Tampoco forman parte de la accesibilidad las prácticas traductológicas e interpretativas comunes, generales. Traducir a Shakesperare facilita el acceso a su obra, sin que por ello podamos hablar en este caso de accesibilidad. Aunque podría discutirse, no parece que el destinatario de la práctica traductológica sea aquí un colectivo significado en términos de diversidad social y con riesgo de exclusión social.

C. El espacio de intersección del diagrama anterior trata de delimitar precisamente ese dominio en el que entendemos que accesibilidad se define como práctica de la mediación intercódigo, y viceversa. Este subespacio de 
intersección no está represado por un práctica única y homogénea. Debemos entender que se diversifica en múltiples sentidos. Pero hay al menos dos sentidos genéricos que conviene destacar.

C1. En buena parte de los estudios que vinculan académicamente el análisis de la accesibilidad con estudios de traducción e interpretación, el objetivo que se plantea es una labor facilitadora del proceso comunicativo dentro de lo que Jakobson (1959) denominaba la traducción intralingüística. En este último sentido, accesibilidad se entiende como actividad reformuladora. Se ha vinculado especialmente la accesibilidad al ámbito de la traducción audiovisual, y concretamente a labores como la de la audiodescripción para ciegos y la subtitulación para sordos (Orero 2007). En este caso no habría en realidad actividad de traducción si por ella entendemos cambio de sistema lingüístico, pero parece asumido desde hace tiempo que la traducción puede definirse como reescritura (Lefevere 1992) y, aunque no es exactamente lo mismo, los procesos de reescritura pueden formar parte de lo que un traductor o intérprete realiza en su actividad profesional. Una lengua no es una realidad uniforme sino un conjunto de códigos en convivencia entre los cuales nos vemos muchas veces obligados a mediar. Esta vinculación a la traducción audiovisual no es, sin embargo, sustancial en la delimitación del concepto de accesibilidad. Una reformulación guiada por el objetivo de la accesibilidad no necesariamente se restringe al ámbito de los medios audiovisuales. Por ejemplo, dentro del marco de las patologías del lenguaje se han desarrollado programas informáticos para la simplificación sintáctica de textos (Carol et al. 1998), lo que facilita su lectura a colectivos que presentan dificultades en el proceso de comprensión lectora debidas a daño neurológico. Parece claro que este tipo de práctica forma parte de una accesibilidad donde se implican los procesos comunicativos.

C2. Nos preguntamos ahora, dentro de este subespacio de intersección, dónde confluyen accesibilidad y una práctica de mediación interlingüística propiamente dicha, esto es, una mediación intercódigo cuando los códigos implicados son dos sistemas lingüísticos diferenciados. La respuesta nos lleva aquí directamente sobre la práctica de la interpretación social, también interpretación para servicios públicos o "community interpreting" en la formulación inglesa (Carr 1997, Hale 2007, Martín 2000, Valero-Garcés \& Martín 2008). 
En lo que sigue nuestra idea será aportar los argumentos por los cuales la interpretación social no es un dominio más que se suma al paraguas académico de la accesibilidad, sino una práctica que representa paradigmáticamente la conexión entre accesibilidad y mediación interlingüística. Esta discusión es la que abordamos seguidamente.

\section{Interpretación social como ejemplo paradigmático de una práctica de la accesibilidad}

\subsection{Base metodológica}

En lo que sigue, mi intención es poner de manifiesto una relación de afinidad entre el concepto de accesibilidad y el de interpretación social. Esta relación de afinidad se trata de mostrar estableciendo una correspondencia analógica entre factores definitorios de la accesibilidad y factores también presentes en la práctica de la interpretación social. Para este efecto hemos seleccionado, entre la multiplicidad de propuestas existentes, un estudio sobre el concepto de accesibilidad que ofrece la base teórica para ello y cuyo objetivo más específico se encuentra muy distante de una práctica de la mediación interlingüística, más en concreto: accesibilidad en el diseño de la vivienda. Nos referimos al estudio de Alonso (2007) que lleva el significativo título de "Algo más que suprimir barreras: conceptos y argumentos para una accesibilidad universal", artículo publicado dentro de la revista TRANS. Estudios de Traductología, como parte de un dossier monográfico sobre el tema de la accesibilidad que coordinaba Pilar Orero (Orero 2007).

\subsection{Factor conceptual básico compartido}

Asumiendo lo formulado en la introducción de este estudio, podemos decir que la interpretación social comparte lo que es esencial en el concepto de accesibilidad. En el concepto de accesibilidad se conjuga una actitud de reconocimiento de los factores de diversidad social y una operación encaminada a facilitar la participación en la acción cooperativa humana de colectivos con riesgo de exclusión. Las maneras concretas con las que esta idea general se materializa pueden ser muy variadas. De hecho, la diversidad social no es una foto fija de la que partimos, sino algo en continuo proceso de evolución. Las sociedades son diversas de muchas maneras a lo largo del tiempo. 
Igualmente, el propio reconocimiento de los factores de diversidad va cambiando y depende de los contextos políticos, económicos o culturales. Dada esta realidad "en proceso" que es la diversidad social y su reconocimiento, es fácil entender el carácter también interpretativamente abierto del concepto de accesibilidad. Al interés en una apertura programática del concepto de accesibilidad se refiere Alonso (2007: 16-17) en los siguientes términos:

....en la actualidad también se está reconociendo la implicación que la accesibilidad tiene para la calidad de vida de todas las personas a través de un diseño de mayor calidad y orientado hacia la diversidad de usuarios: cualquier persona debe poder disponer y utilizar con confort y seguridad los entornos, servicios o productos, tanto físicos como virtuales, de forma presencial o no presencial, en igualdad de condiciones que los demás. En consecuencia, la asociación automática de accesibilidad con barreras y discapacidad, debe ser superada para desplegar todo su significado y beneficios en relación con todo tipo de personas.

En las palabras finales de la anterior cita destacamos la idea de que una asociación previa del concepto de accesibilidad al de barreras físicas y discapacidades, aunque justificable, no debe condicionar otras posibles lecturas del término. En palabras del autor, la accesibilidad debe "desplegar todo su significado", lo que supondrá ampliar el marco de los posibles beneficiarios. Asumimos esta visión programática concretándola para el caso de la interpretación social y sus posibles beneficiarios. Quizá más importante que el mero hecho de la inclusión de la interpretación social dentro del concepto de accesibilidad, puede ser el debate o diálogo que se abre al respecto y que nos da justamente una oportunidad para esclarecer el propio concepto de accesibilidad. Proponemos en lo que sigue una serie de analogías entre interpretación social y accesibilidad, partiendo para ello del argumentario que nos propone Alonso (2007).

En cualquier caso, la idea que manejamos es considerar la interpretación social como parte integrante de la accesibilidad. La interpretación social se entiende como instrumento que sirve para la eliminación de las barreras, en este caso, lingüísticas, y que se encuentra especialmente dirigida a extranjeros no hablantes de la lengua del país de acogida. Estos sujetos, como individuos y en tanto que colectivo, presentan potenciales riesgos de exclusión social si 
no se ve facilitada su capacidad de entender y hacerse entender en diversos ámbitos como el laboral, el sanitario, el educativo o el judicial.

\subsection{Analogía respecto al argumento ético-político de la no discriminación}

La accesibilidad, como ya se señalaba en la introducción, se encuentra germinalmente asociada a la noción ilustrada de democracia que reformula el sentido de la cooperatividad humana basándolo en la autonomía del individuo. Esta presencia germinal del concepto de accesibilidad ha tenido que desarrollarse a lo largo del tiempo y el recorrido no ha estado por supuesto exento de esfuerzos y dramas en escenarios de muy variados tipos. Algo, de todas formas, se reprograma mentalmente en el imaginario social democrático, para que lo diverso no se mantenga discriminado, sino que trate de integrarse.

Los partidarios de la esclavitud y, más adelante, de la segregación racial en la primera democracia del mundo, no sintonizaban claramente con el signo de los tiempos. Iban a ser superados sí o sí, pese a su apelación a conductas atávicas que pueden conservar todavía algún respaldo social, y que siempre mantienen un potencial riesgo de revitalización. En el ámbito de la psiquiatría se reconceptualiza la idea de enfermo mental como sujeto que debemos discriminar aislándolo en internados, para buscar en lo posible su integración en el medio social. Algo parecido puede decirse de otros ámbitos del discurso médico. En el caso de las patologías del lenguaje, la terapia pasa de centrarse en el individuo a incluir factores de interacción social. En general, como señala Alonso (2007), se observa un cambio de perspectiva en la visión de la discapacidad que se fue plasmando durante la década de los años 80 del pasado siglo. La idea fundamental es que la deficiencia no es un problema del individuo, con cualquier tipo de discapacidad, sino que afecta al entorno social. En este sentido, el tratamiento de la discapacidad incluiría una respuesta adaptativa del medio social para facilitar la interacción de la persona con discapacidad con su entorno. Alonso (2007: 21) habla de una nueva ética de la discapacidad:

Esta nueva ética de la discapacidad sugiere transformar los entornos de manera que resulten más sencillos, cómodos y seguros para que todas las personas puedan desarrollar en ellos una vida activa, autónoma e independiente. 
En realidad, esta nueva "ética de la discapacidad" no deja de ser sino una manifestación particular del argumento político general de la no discriminación y la inclusión participativa de lo diferente. Esta nueva perspectiva ética habría generado una aparato normativo bajo el lema de la no discriminación y la igualdad de oportunidades. Muestra de ello es el artículo 13 del Tratado de Ámsterdam de 1996:

Sin perjuicio de las demás disposiciones del presente Tratado y dentro de los límites de las competencias atribuidas a la Comunidad por el mismo, el Consejo, por unanimidad, a propuesta de la Comisión y previa consulta al Parlamento Europeo, podrá adoptar acciones adecuadas para luchar contra la discriminación por motivos de sexo, de origen racial o étnico, religión o convicciones, discapacidad, edad u orientación sexual.

Más específico respecto a una política inclusiva referida a la discapacidad resulta la Declaración de Estocolmo, del European Institute for Design and Disability, cuya versión en español me permito también reproducir aquí:

Como consecuencia de todo ello, el European Institute for Design and Disability, en su Junta Anual celebrada en Estocolmo el 9 de Mayo del 2004, aprobó la siguiente Declaración:

En Europa, la diversidad humana ha aumentado en cuanto a la edad, la cultura y la capacidad. Actualmente superamos enfermedades y lesiones y por lo tanto convivimos con más discapacidades. A pesar de que el mundo de hoy es un lugar complejo, lo hemos construido nosotros, por lo tanto, tenemos la posibilidad y la responsabilidad de basar nuestros diseños en los principios de inclusión.

El Design for All es el diseño que tiene en cuenta la diversidad humana, la inclusión social y la igualdad. Este acercamiento holístico e innovador constituye un reto creativo y ético para todos los responsables de la planificación, el diseño, la gestión y la administración, así como para los políticos.

El Design for All tiene como objetivo hacer posible que todas las personas dispongan de igualdad de oportunidades y de participar en cada aspecto de la sociedad. Para conseguir esto, el entorno construido, los objetos cotidianos, los servicios, la cultura y la información, en resumen, todo lo que está diseñado o hecho por personas para las personas ha de ser accesible, y útil para todos los miembros de la sociedad y consecuente con la continua evolución de la diversidad humana.

La practica del Design for All conlleva también el análisis de las necesidades y las expectativas humanas y requiere la participación de los usuarios en todas las fases del diseño. 
Por lo tanto, el European Institute for Design and Disability insta a las instituciones europeas, nacionales, regionales y a los gobiernos locales, profesionales, empresas y otros actores sociales a tomar todas las medidas apropiadas para implantar el Design for All en sus políticas y acciones.

Aprobada el 9 de Mayo del 2004, en la Junta Anual del European Institute for Design and Disability, en Estocolmo.

Declaraciones de este tipo podemos encontrar también en organismos nacionales, pero todas ellas están inspiradas en el principio ético-político de la no discriminación, y se encuentran orientadas a la adaptación de espacios, productos y servicios que permiten la plena participación activa de los sujetos con discapacidad en su entorno social.

¿En qué medida la interpretación social responde a este argumento ético-político de la no discriminación? Es obvio que lo hace de manera central. La interpretación social suele implicar sujetos cuya condición de extranjero habitualmente se asocia al desconocimiento lingüístico y la incapacidad de comunicación. Constituyen una clara muestra de esa diversidad social que debe reconocerse ética y normativamente. A esta diversidad contribuyen al mismo tiempo factores raciales, culturales, religiosos y también específicamente lingüísticos. Queda, sin embargo, mucha labor pendiente de "pedagogía social" en este caso concreto.

Álvarez Gálvez (2009) señala, por ejemplo, en el caso español cómo el tipo de encuadre de las noticias periodísticas o televisivas sobre la inmigración se asocia implícitamente a la idea de peligro o amenaza para la sociedad receptora. El modo en que se presentan las noticias contribuye a la estigmatización del inmigrante, con el consiguiente efecto segregador que ello supone. Este efecto negativo de los medios respecto a nuestra visión del inmigrante es señalado también por otros muchos autores (Bañón 1996, Granados 1998, Rodrigo Alsina 1999, Van Dijk 1997, etc.). Álvarez Gálvez (2009), asumiendo ese papel de construcción social de los medios de comunicación, aboga justamente por una redefinición de la política informativa que asuma también para este colectivo el principio ético-político de la no discriminación. En las conclusiones de su estudio formula lo siguiente:

Ahora bien, partiendo de la propia capacidad de los medios para construir la realidad, conviene hacer referencia a las posibilidades de estos de cara a la extinción del racismo y la xenofobia de nuestras sociedades. Efectivamente, 
del mismo modo que los medios de comunicación tienen capacidad para acrecentar los problemas, también pueden favorecer la erradicación o minimización de estos. Asimismo, al igual que pueden incrementar los sentimientos de rechazo hacia los inmigrantes, también pueden fomentar los sentimientos de tolerancia y aceptación mediante un tratamiento positivo (Calvo Buezas, 1996). En este sentido, se hace manifiesta la necesidad de una ética mediática que permita y favorezca un tratamiento más cuidadoso de la inmigración como grupo en riesgo de exclusión. (Alvárez Gálvez 2009: 77-78)

Ciertamente, no solo los medios de comunicación, sino también otros agentes sociales, como las instituciones educativas en particular, deben contribuir a la acción de este principio político de la no discriminación en el caso particular del colectivo (o los colectivos) de inmigrantes.

\subsection{Analogía respecto al argumento legal/normativo}

El reflejo del principio general de la no discriminación, y la búsqueda de cauces para una integración participativa de colectivos con riesgo de exclusión social, se va abriendo paso muy poco a poco en el aparato normativo o legal. Entre las declaraciones ético-políticas y el reflejo legal o normativo, hay un "décalage" temporal bastante significativo. Esta situación es algo que puede referirse tanto al tema de la accesibilidad en general como al caso particular de la interpretación social. Debe reconocerse de todas formas que la reglamentación relativa a la eliminación de barreras físicas y determinadas situaciones discapacidad sensorial se adelantan en lo referido a reglamentos concretos sobre otras situaciones. Hay cierta disparidad explicable en lo referido a la reglamentación de la accesibilidad dependiendo de los diferentes ámbitos de aplicación.

Para la interpretación social podemos decir, en general, que hay un vacío legal en España (no tanto en otros países). Solo de manera implícita el artículo 9 de la Constitución Española, contiene un mandato relativo a la accesibilidad. El artículo dice lo siguiente en su parágrafo 2:

Artículo 9. 2. Corresponde a los poderes públicos promover las condiciones para que la libertad y la igualdad del individuo y de los grupos en que se integra sean reales y efectivas; remover los obstáculos que impidan o dificulten su plenitud y facilitar la participación de todos los ciudadanos en la vida política, económica, cultural y social. 
El artículo 49 es algo más explícito en lo relativo a la regulación de la accesibilidad, aunque el tema de las barreras comunicativas no se menciona ni aquí, ni en ningún otro artículo de la Constitución:

Artículo 49. Los poderes públicos realizarán una política de previsión, tratamiento, rehabilitación e integración de los disminuidos físicos, sensoriales y psíquicos, a los que prestarán la atención especializada.

La accesibilidad comunicativa es algo, sin embargo, especialmente focalizado en el ámbito judicial. La exigencia de una plasmación normativa deriva del Artículo 10 de la Declaración Universal de los Derechos Humanos, en el que se formula lo siguiente:

Toda persona tiene derecho, en condiciones de plena igualdad, a ser oída públicamente y con justicia por un tribunal independiente e imparcial, para la determinación de sus derechos y obligaciones o para el examen de cualquier acusación contra ella en materia penal.

En consonancia con lo que se dice en este artículo, y atendiendo a las especiales circunstancias que imponen las barreras comunicativas, la Ley de Enjuiciamiento Criminal española somete a regulación la figura y la actividad de un traductor o intérprete judicial. Como se muestra en el apartado 3 del artículo 124 de esta Ley, junto a las barreras que imponen los idiomas se citan también las dificultades comunicativas de "las personas sordas o con discapacidad auditiva". De esta forma, en la labor de un intérprete judicial se vinculan normativamente barreras idiomáticas y una discapacidad sensorial como la sordera:

Ley de Enjuiciamiento Criminal. Artículo 124.

1. El traductor o intérprete judicial será designado de entre aquellos que se hallen incluidos en los listados elaborados por la Administración competente. Excepcionalmente, en aquellos supuestos que requieran la presencia urgente de un traductor o de un intérprete, y no sea posible la intervención de un traductor o intérprete judicial inscrito en las listas elaboradas por la Administración, en su caso, conforme a lo dispuesto en el apartado 5 del artículo anterior, se podrá habilitar como intérprete o traductor judicial eventual a otra persona conocedora del idioma empleado que se estime capacitado para el desempeño de dicha tarea.

2. El intérprete o traductor designado deberá respetar el carácter confidencial del servicio prestado. 
3. Cuando el Tribunal, el Juez o el Ministerio Fiscal, de oficio o a instancia de parte, aprecie que la traducción o interpretación no ofrecen garantías suficientes de exactitud, podrá ordenar la realización de las comprobaciones necesarias y, en su caso, ordenar la designación de un nuevo traductor o intérprete. En este sentido, las personas sordas o con discapacidad auditiva que aprecien que la interpretación no ofrece garantías suficientes de exactitud, podrán solicitar la designación de un nuevo intérprete.

La Unión Europea es también normativamente explícita con el derecho a la interpretación y la traducción en procesos penales. Dentro de la Directiva 64/2010 de la UE, se afirma lo siguiente en su artículo 2:

1. Los Estados miembros velarán por que todo sospechoso o acusado que no hable o entienda la lengua del proceso penal se beneficie sin demora de interpretación en el transcurso del proceso penal ante las autoridades de la investigación y judiciales, incluido durante el interrogatorio policial, en todas las vistas judiciales y las audiencias intermedias que sean necesarias. 2. Los Estados miembros garantizarán que, en caso necesario y con miras a salvaguardar la equidad del proceso, se facilite un servicio de interpretación para la comunicación entre el sospechoso o acusado y su abogado en relación directa con cualquier interrogatorio o toma de declaración durante el proceso, o con la presentación de un recurso u otras solicitudes procesales. 3. El derecho a interpretación en virtud de los apartados 1 y 2 incluye la asistencia a personas con limitaciones auditivas o de expresión oral.

Aunque la previsión de asistencia es genérica, la normativa está especialmente dirigida a población inmigrante con potencial riesgo de exclusión social. Se delega en las correspondientes autoridades judiciales la valoración sobre la necesidad de asistencia, entendiéndose que casos particulares no deberían suscitar muchas dudas a la jurisprudencia. Es importante subrayar el hecho de que se asimilen también aquí, según se dice en el apartado 3 de la anterior cita, las situaciones de desconocimiento de lenguas, con la de "limitaciones auditivas" o de "expresión oral". Queda en este sentido clara la conexión que en la normativa para la accesibilidad se debe establecer entre barreras de código lingüístico y barreras de tipo sensorial que limitan la capacidad comunicativa. La normativa contempla también la necesidad de acreditación para el desempeño de las labores de intérprete, tal como figura en el artículo 5 sobre la "calidad de la traducción y la interpretación": 
1. Los Estados miembros tomarán medidas para garantizar que la interpretación y la traducción facilitadas se ajusten a la calidad exigida con arreglo al artículo 2, apartado 8, y el artículo 3, apartado 9.

2. Con objeto de fomentar la idoneidad de la interpretación y traducción, así como un acceso eficaz a las mismas, los Estados miembros se esforzarán por establecer uno o varios registros de traductores e intérpretes independientes debidamente cualificados. Una vez establecidos dichos registros se pondrán, cuando proceda, a disposición de los abogados y las autoridades pertinentes. 3. Los Estados miembros garantizarán que los intérpretes y traductores respeten el carácter confidencial inherente a los servicios de interpretación y traducción facilitados de conformidad con la presente Directiva.

Esta regulación normativa en el ámbito de la interpretación judicial debería desarrollarse también en otros, como la interpretación social en el ámbito médico, el laboral o el educativo, con las consiguientes implicaciones en la profesionalización de los intérpretes sociales. Algo se va avanzando en este sentido, pero entendemos que, dadas las implicaciones particulares de los procesos judiciales y lo que pueden comportar las sentencias para sus implicados, el ámbito judicial haya tenido prioridad normativa sobre otros ámbitos de la interpretación social.

\subsection{Analogía respecto al argumento demográfico}

Parece claro que una valoración cuantitativa de la discapacidad depende en gran medida del criterio cualitativo que manejamos a la hora de determinar esta condición, y los tipos y grados con los que se manifiesta una situación de dependencia. Ello explica, por ejemplo, las disparidades cuantitativas que nos muestra Alonso (2007) en una gráfica donde se recogen estadísticas por países. Las diferencias se pueden atribuir sin duda en gran medida a diferentes maneras de conceptualizar la condición de discapacitado. La incidencia de determinados tipos de discapacidad como la ceguera o la sordera pueden ser porcentualmente estables a lo largo del tiempo, con matices. Pero en otros casos, como por ejemplo la demencia senil, o colectivos de personas que sobreviven con secuelas a diferentes tipos de lesiones físicas o neurológicas, el número de personas implicadas se ha ido acrecentando con el envejecimiento general de las sociedades actuales y con los avances médicos que hacen posible la supervivencia tras accidentes de variado tipo. Estas situaciones son 
valoradas de forma muy diferente en lo que se refiere a la condición de dependiente para la persona implicada.

En lo que se refiere a la interpretación social el colectivo afectado en el contexto europeo y en el español es cada vez más significativo. Es obvio que ha existido un incremento muy notable de la población inmigrante durante la dos últimas décadas en Europa y en España. Eso se traduce en un incremento también de la perceptibilidad social del fenómeno. Por lo que se refiere en particular al caso español, el incremento ha sido progresivo y muy apreciable desde principios de los años 90 hasta el inicio de la crisis financiera de 2008 y la recesión económica global que comportó. De hecho, como se observa en la tabla (datos obtenidos del Instituto Nacional de Estadística), la población global de inmigrantes, refiriéndonos aquí a la procedente de países de habla no española, ha decrecido desde 2012 hasta 2018, pero se mantiene en torno a los dos millones y medio de personas.

\begin{tabular}{|l|r|r|r|r|}
\hline \multicolumn{1}{|c|}{ País } & \multicolumn{1}{c|}{2018} & \multicolumn{1}{c|}{2012} & \multicolumn{1}{c|}{2001} & \multicolumn{1}{c|}{2000} \\
\hline Totales & 2.388 .842 & 2.654 .110 & 407.860 & 229.138 \\
\hline Marruecos & 769050 & 788563 & 233415 & 173158 \\
\hline Rumania & 673017 & 897137 & 31641 & 6410 \\
\hline China & 215748 & 175813 & 27574 & \\
\hline Bulgaria & 123730 & 176216 & 12035 & \\
\hline Ucrania & 106823 & 88438 & 10318 & \\
\hline Pakistán & 82738 & 79626 & 8274 & 4195 \\
\hline Brasil & 81464 & 99043 & 17078 & 11126 \\
\hline Rusia & 73700 & 57275 & 10047 & 5199 \\
\hline$\underline{\text { Senegal }}$ & 66046 & 63491 & 10627 & \\
\hline Argelia & 60820 & 62432 & 18265 & 10759 \\
\hline Polonia & 52212 & 84197 & 13469 & 8164 \\
\hline India & 43520 & 35888 & 7996 & 6807 \\
\hline Nigeria & 39374 & 45991 & 7598 & 3320 \\
\hline
\end{tabular}

Hablamos de un número de personas para las que las barreras comunicativas pueden presentar y presentan de hecho un riesgo notable de exclusión social. 
Por tener un dato comparativo, pensemos que, según datos del Instituto Nacional de Estadística, el número global de discapacitados se estimaba en 2009 en torno a los tres millones y medio de personas. Este número incluye todo tipo de discapacidad reconocida como tal, aunque el requerimiento de políticas de accesibilidad para este colectivo puede ser muy diferente. Si consideramos un colectivo particular, como el de los casos de ceguera, según Gómez Ulla (2012) el número de personas con ceguera total en España representaba en 2008 un colectivo de 58.300 personas, si bien los casos muy variados de baja visión alcanzaba el número de 920.900. La sordera se manifiesta también con muy diferentes grados de severidad, pero el colectivo con algún tipo de discapacidad auditiva ronda también el millón de personas según datos del INE. No todos los casos de déficit visual o auditivo deben considerarse, sin embargo, objetivo de las políticas de accesibilidad.

Si admitimos que, conceptualmente, las barreras lingüísticas pueden ser factores implicados en la discriminación social para el colectivo inmigrante, está claro que en términos meramente cuantitativos merecen un especial foco de atención. El incremento de población inmigrante vivido en las últimas décadas incrementa también sin duda, como se ha dicho, la toma de conciencia social sobre el fenómeno y pone de manifiesto la necesidad de ampliar el concepto de accesibilidad al mismo.

\subsection{Analogía respecto al argumento económico}

Al margen de beneficios que tienen que ver con la satisfacción de necesidades individuales, se puede plantear el tema de la accesibilidad en términos incluso de rentabilidad económica. Como se proponía en la introducción de este estudio, la accesibilidad no es un simple servicio que se ofrece, sino un instrumento guiado por el principio de cooperación social. En este sentido, las políticas de accesibilidad contribuyen también globalmente a la actividad económica de un país en la medida en que incrementan la actividad cooperativa entre los miembros de una sociedad. Por mencionar un ejemplo, el subtitulado para sordos incrementa el número de consumidores de los productos audiovisuales. Facilitar acceso físico puede permitir que personas especialmente capacitadas para el ejercicio de una profesión puedan ejercerla, y contribuir al desarrollo social. Etc. 
En el caso de la interpretación social dirigida al colectivo de inmigrantes los beneficios en términos de rentabilidad económica son particularmente obvios. La inmigración constituye un capital humano del que la sociedad europea y en particular la española no pueden prescindir. En un informe sobre inmigración del Consejo Económico y Social de España (2019: 186) se afirma:

La incorporación de gran número de inmigrantes en edad laboral al mercado de trabajo español fue una pieza clave del modelo productivo que caracterizó el periodo de expansión de la economía española hasta el inicio de la crisis en 2008. El patrón de crecimiento español se basó en la expansión de sectores intensivos en mano de obra, singularmente la construcción, pero también algunos servicios como el comercio y la hostelería, donde los inmigrantes se fueron integrando, cada vez en mayor número, debido a la insuficiencia de los incrementos de población activa autóctona para cubrir la incesante demanda de trabajo que generaban estos sectores (...) Según evidencian diversos estudios, este significativo aumento de la población activa extranjera no tuvo efectos negativos ni sobre el empleo ni sobre los niveles salariales de la población autóctona.

Un aspecto particular que se destaca en este informe es el hecho de que la contribución de la población inmigrante a la atención doméstica y justamente también en tareas relacionadas con situaciones de dependencia, "contribuyó en gran medida a la expansión de la tasa de actividad de las mujeres españolas cualificadas con responsabilidades familiares que caracterizó el periodo de expansión económica" (Consejo Ecónomico y Social de España 2019: 187). Si nos referimos al capítulo de gastos, y frente a lo que pueden ser opiniones no justificadas, el gasto médico asociado a la población inmigrante es en términos porcentuales inferior al de la población autóctona, según el mencionado informe. En definitiva, directa o indirectamente, la población inmigrante supone una contribución muy notable al producto interior bruto del país. Las políticas de inclusión y accesibilidad para ese colectivo se encuentran, así pues, justificadas en términos económicos, al margen de los argumentos ético-políticos.

\section{Conclusiones}

Las conclusiones que derivan de todo lo anterior parecen claras. Las analogías que se han presentado nos permiten afirmar que las labores propias de un intérprete social pueden, y tal vez deben, conceptualizarse en términos de 
accesibilidad. Los paralelismos que hemos observado respecto a las consideraciones nocionales, éticas, políticas, normativas, de número de personas afectadas y, finalmente, económicas avalan perfectamente la propuesta que se hace de conexión disciplinar entre accesibilidad e interpretación social.

Reconocemos, sin embargo, que en los estudios traductológicos accesibilidad e interpretación social han constituido hasta el momento dominios de investigación diferenciados. Puede ser que, por motivaciones académicas de diferente tipo, lo sigan estando. Pero espero que la propuesta de conexión disciplinar que se formula en este estudio, haya contribuido al menos a esclarecer el "concepto" genérico de accesibilidad, con independencia del ámbito concreto que convencionalmente se designa con el "término" accesibilidad.

\section{Referencias}

AlONSO, Armin Paul; Kurt-Jürgen Maass; Fritz Paul \& Horst Turk (eds.) (1993) Übersetzen, verstehen, Brücken bauen. Berlin: Erich Schmidt.

AlOnSO, Fernando. (2007) "Algo más que suprimir barreras: conceptos y argumentos para una accesibilidad universal." Trans. Revista de Traductología 11, pp. 15-30.

ÁlvAREZ GÁlVEZ, Javier. (2009) "La representación mediática de la inmigración. Entre el encuadre y el estigma." Revista del Ministerio de Trabajo e Inmigración 80, pp. 61-80.

BAÑón, Antonio M. (1996) Racismo, discurso periodístico y didáctica de la lengua. Almería: Universidad de Almería.

Caroll, John; Guido Minnen; Yvonne Canning; Siobhan Devlin \& John Tait. (1998) "Practical simplification of English newspaper text to assist aphasic readers." En: Proceedings of AAAI98 Workshop on Integrating Artificial Intelligence and Assistive Technology. Madison, Wisconsin.

CONSEJO ECONÓMICO Y SOCIAL DE ESPAÑA. (2019) Informe sobre la inmigración en España: Efectos y oportunidades. Versión electrónica: <http://www.ces.es/ documents/10180/6053176/Inf0219.pdf>

Granados, Antolín. (1998) La imagen del inmigrante extranjero en la prensa española: ABC, Diario 16, El Mundo y El País (1985-1992).Tesis Doctoral: Universidad de Granada.

GómeZ-Ulla, F. (coor.) 2012. Informe sobre la ceguera en España. Fundación Retinaplus+ y Ernst \& Young. 
HALE, Sandra. (2007) Community Interpreting. Basingstoke (RU): Palgrave Macmillan.

Hurtado Albir, Amparo. (1990) La notion de fidélité en traduction. Paris: Didier INE. (2009) "Panorámica de la discapacidad en España". Cifras INE. Boletín informativo del Instituto Nacional de Estadística.

JAKOBSON, Roman. (1959) "On Linguistic Aspects of Translation”. En: Brower, Reuben A. (ed.) 1959. On Translation. Cambridge Mass: Harvard University Press, pp. 232-239.

LEFEVERE, André. (1992) Translation, Rewriting, and the Manipulation of Literary Fame. London: Routledge.

MARTin, Anne. (2000) "La interpretación social en España." En: Kelly, Dorothy (ed.) 2000. La traducción y la interpretación en España hoy: Perspectivas profesionales. Granada: Comares, pp. 207-223.

Martin, Anne. (2003) "Investigación en interpretación social: Estado de la cuestión." En: Ortega Arjonilla, Emilio (dir.) 2003. Panorama actual de la investigación en traducción e interpretación, (2 vols.), vol. I. Granada: Atrio, pp. 431-446.

MATUSALL, Svenja. (2013) "Social behavior in the "Age of Empathy"?-A social scientist's perspective on current trends in the behavioral sciences." Frontiers of Human Neuroscience 31 May 2013.

ORERO, Pilar. (2007) "La accesibilidad en los medios: una aproximación multidisciplinar." Trans. Revista de Traductología 11, pp. 11-14.

SACKS, Oliver. (2006) Veo una voz. Viaje al mundo de los sordos. Barcelona: Anagrama.

Rodrigo Alsina, Miquel. (1999) Comunicación intercultural. Barcelona: Anthropos.

VAlero GarCÉs, Carmen. (2003) "Una visión general de la evo- lución de la traducción e interpretación en los servicios públicos." En: Valero Garcés, Carmen (ed.) 2003. Traducción e interpretación en los Servicios Públicos. Contextualización, actualidad y futuro. Granada: Comares, Colección Interlingua, pp. 3-33.

VAlero-Garcés, Carmen \& Anne Martin (eds.) (2008) Crossing Borders in Community Interpreting. Definitions and Dilemmas. Ámsterdam \& Filadelfia: John Benjamins.

VAN Dijk, Teun A. (1997) Racismo y análisis crítico de los medios. Barcelona: Paidós. 


\section{BIONOTE / BIONOTA}

SAIDA ANSSARI-NAIM is a lecturer in Translaltion and Interpreting at the University of Valencia. She has studied at the University of Fez (Morocco), La Sorbonne (Paris IV), and the University of Valencia. Her research is centred on the problems of cross-cultural communication from a linguistic-anthropology perspective. She has collaborated in European research projects on Migration and Development. She is currently a member of a research project on translation, interpreting and cognition. She is an official translator of Arabic. She is a native speaker of Arabic and French and is also proficient in English, Spanish and Catalan. She has recently published a book in the "English in the World Series" (PUV): The Politeness/Impoliteness Divide. English-Based Theories and Speech Acts Practice in Moroccan Arabic, Valencia, 2016. She has recently published several articles on the cognitive aspects of interpreting.

SAIDA ANSSARI-NAIM es profesora Ayudante Doctor de Traducción e Interpretación en la Universidad de Valencia. Ha estudiado en la Universidad de Fez (Marruecos), La Sorbona (París IV) y la Universidad de Valencia. Su investigación ha atendido aspectos de la comunicación intercultural desde una perspectiva lingüístico-antropológica. Ha colaborado en proyectos de investigación europeos sobre migración y desarrollo. Actualmente es miembro de un proyecto de investigación ministerial dentro del cual desarrolla una línea de investigación en traducción, interpretación y cognición. Es traductora oficial de árabe. Es hablante nativa de árabe y francés y también domina el inglés, el castellano y el catalán. Es autora del libro: The Politeness/Impoliteness Divide. English-Based Theories and Speech Acts Practice in Moroccan Arabic, Valencia, 2016. Ha publicado recientemente varios artículos sobre los aspectos cognitivos de la interpretación. 